\title{
Genetic associations in the detection of QTLs for wheat spike-related traits
}

\author{
Ljiljana Brbaklić(1), Dragana Trkulja( ${ }^{(1)}$, Ankica Kondić-Špika(1), Nikola Hristov ${ }^{(1)}$, Srbislav Denčić(1), \\ Sanja Mikić(1), Marina Tomičić(1) and Borislav Kobiljski(2)
}

${ }^{(1)}$ Institute of Field and Vegetable Crops, Maksima Gorkog 30, 21000 Novi Sad, Serbia. E-mail: ljiljana.brbaklic@nsseme.com,
dragana.trkulja@nsseme.com, ankica.spika@nsseme.com, nikola.hristov@nsseme.com, srbislav.dencic@ifvcns.ns.ac.rs,
sanja.mikic@nsseme.com, marina_tomicic@yahoo.com ${ }^{(2)}$ Biogranum, Toplice Milana 20, 21000 Novi Sad, Serbia. E-mail: kobboris@gmail.com

Abstract - The objective of this work was to assess the genetic diversity and population structure of wheat genotypes, to detect significant and stable genetic associations, as well as to evaluate the efficiency of statistical models to identify chromosome regions responsible for the expression of spike-related traits. Eight important spike characteristics were measured during five growing seasons in Serbia. A set of 30 microsatellite markers positioned near important agronomic loci was used to evaluate genetic diversity, resulting in a total of 349 alleles. The marker-trait associations were analyzed using the general linear and mixed linear models. The results obtained for number of allelic variants per locus (11.5), average polymorphic information content value (0.68), and average gene diversity (0.722) showed that the exceptional level of polymorphism in the genotypes is the main requirement for association studies. The population structure estimated by model-based clustering distributed the genotypes into six subpopulations according to log probability of data. Significant and stable associations were detected on chromosomes 1B, 2A, 2B, 2D, and 6D, which explained from 4.7 to $40.7 \%$ of total phenotypic variations. The general linear model identified a significantly larger number of marker-trait associations (192) than the mixed linear model (76). The mixed linear model identified nine markers associated to six traits.

Index terms: Triticum aestivum, genetic resources, microsatellites, population structure, spike-related traits.

\section{Associação genética na detecção de QTLs relacionados a características da espiga de trigo}

Resumo - O objetivo deste trabalho foi avaliar a diversidade genética e a estrutura de população de genótipos de trigo, para detectar associações genéticas significativas e estáveis, bem como avaliar a eficácia de modelos estatísticos para identificar as regiões cromossômicas responsáveis pela expressão de características da espiga. Foram determinadas oito importantes características durante cinco safras agrícolas na Sérvia. Uma série de 30 marcadores microssatélites, localizados próximos a locos agronomicamente importantes, foi utilizada para avaliação da diversidade genética, o que resultou num total de 349 alelos. As associações marcador-características foram analisadas com uso dos modelos linear generalizado e linear misto. Os resultados obtidos para número de variantes alélicas por loco $(11,5)$, valor médio de conteúdo de informação polimórfica $(0,68)$ e diversidade genética média $(0,722)$ mostraram que o nível excepcional de polimorfismo nos genótipos é o principal requerimento para estudos de associação. A estrutura da população estimada pelo agrupamento com base no modelo distribuiu os genótipos em seis subpopulações, de acordo com o log da probabilidade dos dados. Associações significativas e estáveis foram detectadas nos cromossomos 1B, 2A, 2B, 2D e 6D, que explicaram de 4,7 a $40,7 \%$ do total das variações fenotípicas. O modelo linear generalizado revelou número significativamente maior de associações marcador-características (192) do que o modelo linear misto (76). O modelo linear misto identificou nove marcadores associados a seis características.

Termos para indexação: Triticum aestivum, recursos genéticos, microssatélites, estrutura da população, características da espiga.

\section{Introduction}

Wheat (Triticum aestivum L.) breeders worldwide invest a great deal of effort into creating cultivars able to challenge rising global issues, such as ongoing climate changes and a growing world population. A tendency in the breeding process is introducing 
novel techniques and approaches that could improve current conventional breeding programs. Particularly, the advancement in the field of molecular biology by applying genetic marker technologies and new statistical approaches are powerful tools for indirect selection of valuable traits through marker-assisted selection (Landjeva et al., 2007). The detection of specific and precisely tagged chromosome regions responsible for the expression of certain agronomic traits could be an excellent contribution for the selection and generation of new high-yielding wheat varieties. Likewise, the knowledge of population diversity and structure is of major importance for an efficient use of elite lines and varieties in a breeding process (Laido et al., 2013).

Spike-related traits are important yield components, which are less environmentally sensitive and exhibit higher heritability than yield per se (Cuthbert et al., 2008). The analyses of the genetic control of spike-related characteristics and of individual effects of different genes and quantitative trait loci (QTL) could provide specific information and be useful for indirect determination of yield improvement (Ma et al., 2007). In the last few years, association mapping has been considered one of the most promising methods for the exploration of the entire genome in the search of preferred chromosome regions, QTLs, and desired genes (Liu et al., 2010). The association mapping approach provides a greater potential for the identification of targeted QTLs and fine tuning and mapping of genes at a higher resolution than the previously used linkage mapping. Based on linkage disequilibrium, association mapping is applied directly to diverse genetic materials, resulting in a larger number of detected alleles per locus in a more representative genetic background. It also represents a higher resolution system due to the recombination events that have been accumulated during selection circles through evolution and historical breeding processes (Haseneyer et al., 2010). Cultivars genotyped with high-density markers and their associations show promise in resolving the genetic basis of complex traits of agronomic and economic importance (Wang et al., 2012). The analysis of complex traits by association mapping is required for breeders, since it facilitates even more the application of associated markers in the breeding process. One of the first association mapping studies in wheat aimed at identifying significant markers for kernel size and milling quality (Breseghello
\& Sorrells, 2006). Subsequently, a large number of works used genome-wide association studies (GWAS) to detect marker-trait associations (MTAs) for a large number of traits, including quality traits in soft wheat (Reif et al., 2011), yield and other agronomic traits in wheat (Liu et al., 2010), and seed longevity in hexaploid wheat (Rehman Arif et al., 2012). In bread wheat, a number of yield-component QTLs was associated with spike-related and adaptive traits (Neumann et al., 2011). The Tassel software (Bradbury et al., 2007) is one of the most sophisticated software programs with implemented algorithms and methods useful for association studies. The structure association analysis developed by Pritchard et al. (2000) first uses a set of random markers to estimate the population structure (Q matrix) and then incorporates this estimation into a general linear model (GLM) analysis. Yu et al. (2006) developed a new methodology, the mixed linear model (MLM) method, which incorporates both the population structure and the familial relatedness or the so-called "kinship" (K matrix), adapted for GWAS, to avoid false associations. This method is recommended in the absence of available pedigree data for clustering a large dataset into groups with improved statistical power (Zhang et al., 2010).

The objective of this work was to assess the genetic diversity and population structure of wheat genotypes, to detect significant and stable genetic associations, as well as to evaluate the efficiency of statistical models to identify chromosome regions responsible for the expression of spike-related traits.

\section{Materials and Methods}

A set of 283 wheat accessions originating from 24 countries was used for phenotype evaluation (Table 1). These varieties are part of the largest Wheat Core Collection in Serbia, which belongs to the Small Grains Department of the Institute of Field and Vegetable Crops in Novi Sad. The genotypes were sown in a randomized complete block design in a $1.2 \mathrm{~m}^{2}$ plot, containing six rows, with a distance of $20 \mathrm{~cm}$ between rows. Field plots were cultivated at Rimski Šančevi $\left(45^{\circ} 20^{\prime} \mathrm{N}, 1^{\circ} 51^{\prime} \mathrm{E}\right)$ in Novi Sad, Serbia, by applying standard agrotechnical practices (Malešević et al., 1994). The following spike-related traits were measured and recorded for association analysis, during five growing seasons, from 1995 to 1999: spike length, 
Table 1. Wheat (Triticum aestivum) varieties and lines, origin, and distribution of subpopulations (genotype clusters, Q) obtained by the Structure software (Pritchard et al., 2000).

\begin{tabular}{|c|c|c|c|c|c|c|c|c|c|c|c|c|c|c|c|}
\hline № & Genotypes & Origin & Q & № & Genotypes & Origin & $\mathrm{Q}$ & № & Genotypes & Origin & $\mathrm{Q}$ & № & Genotypes & Origin & Q \\
\hline 1 & Mironovska264 & Ukraine & 1 & 72 & Pai Yu Pao & China & 5 & 143 & Sremica & Serbia & 5 & 214 & Produttore & Italy & 4 \\
\hline 2 & Stepnjačka30 & Russia & 1 & 73 & San Pastore & Italy & 2 & 144 & Fruškogorka & Serbia & 5 & 215 & Leone & Italy & 5 \\
\hline 3 & Partizanka & Serbia & 5 & 74 & Biserka & Serbia & 1 & 145 & Banaćanka 1 & Serbia & 5 & 216 & 5263 & - & 5 \\
\hline 4 & Una & Serbia & 5 & 75 & NSR2 & Serbia & 5 & 146 & Balkan & Serbia & 5 & 217 & NS $62-20$ & Serbia & 6 \\
\hline 5 & Partizanka niska & Serbia & 5 & 76 & NS736 & Serbia & 1 & 147 & Noe & France & 6 & 218 & NS $62-21$ & Serbia & 6 \\
\hline 6 & Kolubara & Serbia & 5 & 77 & Rana Niska & Serbia & 2 & 148 & NSP 16 & Serbia & 5 & 219 & NS 59-20 & Serbia & 6 \\
\hline 7 & Buckskin & USA & 2 & 78 & Italija & Serbia & 1 & 149 & NS 0.1081 & Serbia & 5 & 220 & NS 59-23 & Serbia & 5 \\
\hline 8 & Tecumseh & USA & 1 & 79 & Kratka & Serbia & 2 & 150 & Žitnica & Serbia & 1 & 221 & Panonija & Serbia & 5 \\
\hline 9 & Bersee0 & France & 2 & 80 & Arg.80/5216 & Argentina & 1 & 151 & Bolonjska & Italy & 5 & 222 & Košava & Serbia & 5 \\
\hline 10 & M.Huntsman0 & $\mathrm{GBR}^{(1)}$ & 2 & 81 & Intro1066 & USA & 2 & 152 & ZG195/7 & Croatia & 4 & 223 & NS 625 & Serbia & 4 \\
\hline 11 & Bersee1 & France & 2 & 82 & NS4/93 & Serbia & 5 & 153 & ZG965 & Croatia & 4 & 224 & NS 974/1 & Serbia & 5 \\
\hline 12 & M.Huntsman1 & GBR & 2 & 83 & NS48/93 & Serbia & 5 & 154 & ZG990 & Croatia & 4 & 225 & NS 984/1 & Serbia & 5 \\
\hline 13 & Tanori71 & Mexico & 2 & 84 & NS90/92 & Serbia & 2 & 155 & ZG1008 & Croatia & 4 & 226 & NS $12-77$ & Serbia & 5 \\
\hline 14 & Lerma Rojo & Mexico & 1 & 85 & NS30/95 & Serbia & 1 & 156 & ZG1020A & Croatia & 4 & 227 & NS $12-87$ & Serbia & 5 \\
\hline 15 & Norteno67 & Mexico & 2 & 86 & Riley & USA & 5 & 157 & ZG8056 & Croatia & 4 & 228 & NS 55-30 & Serbia & 5 \\
\hline 16 & Condor & Australia & 1 & 87 & Knox 62 & USA & 6 & 158 & ZG K 2A/82 & Croatia & 4 & 229 & NS 55-32 & Serbia & 5 \\
\hline 17 & Banks & Australia & 1 & 88 & CombinationN & USA & 3 & 159 & ZG K 77/82 & Croatia & 4 & 230 & NS 56-11 & Serbia & 5 \\
\hline 18 & Aobakomughi & Japan & 1 & 89 & Dimitrovska 5-12 & Bulgaria & 3 & 160 & ZG K 146/82 & Croatia & 4 & 231 & NS 63-15 & Serbia & 5 \\
\hline 19 & Galahad & GBR & 2 & 90 & Purdue $5752 \mathrm{~A}-5-7-2$ & - & 6 & 161 & ZG K 172/82 & Croatia & 4 & 232 & L-44/83 & Serbia & 5 \\
\hline 20 & Dwarf $A^{(2)}$ & GBR & 2 & 91 & $7203-36$ & - & 2 & 162 & ZG K T 171/1/82 & Croatia & 4 & 233 & NS 7003 & Serbia & 3 \\
\hline 21 & Bersee2 & France & 2 & 92 & SI PV 63 & - & 2 & 163 & ZG K 171/1/82 & Croatia & 4 & 234 & NS $7007 / 3$ & Serbia & 5 \\
\hline 22 & M. Huntsman2 & GBR & 2 & 93 & Chinofuz & USA & 2 & 164 & ZG K 176/82 & Croatia & 4 & 235 & ZG 884/73 & Croatia & 3 \\
\hline 23 & Hobbit & GBR & 2 & 94 & $64209-77$ & USA & 2 & 165 & ZG K 242/82 & Croatia & 4 & 236 & ZG 2396/73 & Croatia & 5 \\
\hline 24 & Bounty & GBR & 2 & 95 & Auburn & USA & 6 & 166 & ZG K T 178/82 & Croatia & 4 & 237 & Resistente & Italy & 4 \\
\hline 25 & Sentry & GBR & 2 & 96 & Purdue 79406-I-26-2 & USA & 6 & 167 & Forlani & Italy & 5 & 238 & S.174/72 & - & 5 \\
\hline 26 & Wizard & GBR & 2 & 97 & $\mathrm{NS} 1 / 92$ & Serbia & 6 & 168 & ZG K T 244/82 & Croatia & 4 & 239 & L 60/71 & Serbia & 5 \\
\hline 27 & Norman & GBR & 2 & 98 & ND516 & USA & 3 & 169 & INTRO 7 & USA & 4 & 240 & Fisherect 4A & Mexico & 6 \\
\hline 28 & Mithras & GBR & 2 & 99 & ND517 & USA & 3 & 170 & INTRO 604 & USA & 4 & 241 & Erect Raf E2 & Mexico & 2 \\
\hline 29 & Fenman & GBR & 2 & 100 & Lr1 & USA & 3 & 171 & INTRO 509 & USA & 5 & 242 & UC 66052 & USA & 6 \\
\hline 30 & Sandown & GBR & 2 & 101 & Lr3 & USA & 3 & 172 & INTRO 613 & USA & 4 & 243 & UC 66206 & USA & 6 \\
\hline 31 & Longbow & GBR & 2 & 102 & Lr10 & USA & 3 & 173 & INTRO 29 & USA & 6 & 244 & UC 67052 & USA & 6 \\
\hline 32 & Era & USA & 3 & 103 & Lr11 & USA & 5 & 174 & GSN 17 & USA & 4 & 245 & UC 64246 & USA & 6 \\
\hline 33 & Buckbuck & Mexico & 1 & 104 & Lr12 & USA & 3 & 175 & NSP 54 & Serbia & 5 & 246 & Pudue 6413 & USA & 6 \\
\hline 34 & Olesen Dwarf & Zimbabwe & 1 & 105 & Lr13 & USA & 3 & 176 & NSP 40 & Serbia & 4 & 247 & Sadovo "S" & Bulgaria & 5 \\
\hline 35 & Bersee1+2 & France & 2 & 106 & Lr14 & USA & 3 & 177 & L-63/89 & Serbia & 5 & 248 & Sadovo Super & Bulgaria & 5 \\
\hline 36 & Yecora & Mexico & 1 & 107 & $\operatorname{Lr} 15$ & USA & 3 & 178 & L-64/89 & Serbia & 5 & 249 & M. Dwarf & GBR & 2 \\
\hline 37 & Bersee3 & France & 2 & 108 & Lr16 & USA & 5 & 179 & L-152/89 & Serbia & 5 & 250 & Stephens & USA & 4 \\
\hline 38 & M. Huntsman3 & GBR & 2 & 109 & Lr17 & USA & 3 & 180 & L-154/89 & Serbia & 5 & 251 & Multibraun & Austria & 2 \\
\hline 39 & Bersee2+3 & France & 2 & 110 & Lr20 & USA & 3 & 181 & L-156/89 & Serbia & 5 & 252 & ST 924 (Selekta) & Russia & 4 \\
\hline 40 & Bersee7 & France & 2 & 111 & $\operatorname{Lr} 22$ & USA & 6 & 182 & L-159/89 & Serbia & 5 & 253 & Hilgendorf 61 & Austria & 4 \\
\hline 41 & Akakomughi & Japan & 1 & 112 & Lr30 & USA & 3 & 183 & L-160/89 & Serbia & 5 & 254 & NS 112/92 & Serbia & 5 \\
\hline 42 & Fortunato2D & Italy & 2 & 113 & Tiha & Serbia & 6 & 184 & 30-SC.Smoc.88/89 & Czech Republic & 5 & 255 & Sutjeska & Serbia & 5 \\
\hline 43 & Talent & France & 1 & 114 & NS116/95 & Serbia & 5 & 185 & NS $56 / 90$ & Serbia & 5 & 256 & Zvezda & Serbia & 5 \\
\hline 44 & Cap.dep./Mara & Italy & 2 & 115 & Purdue composite & USA & 6 & 186 & NS $3 / 90$ & Serbia & 6 & 257 & Zelegora & Serbia & 5 \\
\hline 45 & Mara & Italy & 2 & 116 & Purdue 5565 C-4-1-3-3 & USA & 6 & 187 & Vitka & Croatia & 5 & 258 & Szegedi 60 & Hungary & 6 \\
\hline 46 & D6899 & USA & 1 & 117 & Agent & USA & 3 & 188 & Skopljanka & Macedonia & 5 & 259 & WSTGP 91-2 & Hungary & 6 \\
\hline 47 & Cap./Bez.5A & Italy & 2 & 118 & $\mathrm{ABE}$ & USA & 6 & 189 & Nova Skopljanka & Macedonia & 5 & 260 & A.dw.20/6/Ciano 3-5 & - & 4 \\
\hline 48 & S13 & Italy & 1 & 119 & Sap "S"-Mon "S" & USA & 6 & 190 & Radika & Macedonia & 5 & 261 & NSP 192 & Serbia & 6 \\
\hline 49 & NS322 & Serbia & 1 & 120 & OK 75R 3645 & USA & 6 & 191 & Szegedi 5 & Hungary & 4 & 262 & NS $114 / 90$ & Serbia & 5 \\
\hline 50 & NS603 & Serbia & 1 & 121 & T 734-145 & - & 6 & 192 & Lovrin 24 & Romania & 5 & 263 & NS 1/94 & Serbia & 5 \\
\hline 51 & NS732 & Serbia & 1 & 122 & Purdue 77249-RCI-133 & USA & 6 & 193 & Huequen & Chile & 6 & 264 & NS 2/94 & Serbia & 5 \\
\hline 52 & NS900 & Serbia & 2 & 123 & Caldwell & USA & 6 & 194 & Napo 63 & Colombia & 6 & 265 & NS 36/91 & Serbia & 5 \\
\hline 53 & NS $51-11$ & Serbia & 1 & 124 & Pesma & Serbia & 5 & 195 & NO 5519 & China & 5 & 266 & NS 7/94 & Serbia & 4 \\
\hline 54 & NS $54-52$ & Serbia & 1 & 125 & Sreća & Serbia & 5 & 196 & SST 101/A & South Africa & 6 & 267 & NS 9/93 & Serbia & 5 \\
\hline 55 & Bezostaja Dwarf & Russia & 5 & 126 & NS7/93 & Serbia & 5 & 197 & Zarija & Russia & 5 & 268 & NS $18 / 93$ & Serbia & 5 \\
\hline 56 & Pitikul & Moldavia & 5 & 127 & NS83/92 & Serbia & 5 & 198 & PPG-186 & Russia & 5 & 269 & NS $23 / 94$ & Serbia & 5 \\
\hline 57 & Szegedi7610 & Hungary & 2 & 128 & NS22/93 & Serbia & 5 & 199 & Kunčevska & Russia & 5 & 270 & NS 57/92 & Serbia & 5 \\
\hline 58 & Szegedi765 & Hungary & 1 & 129 & NS38/93 & Serbia & 5 & 200 & Nemčinovskaja 110 & Russia & 5 & 271 & L $165 / 94$ & Serbia & 5 \\
\hline 59 & Timson Sun & Australia & 1 & 130 & Adder & USA & 6 & 201 & Triticum spelta ${ }^{(3)}$ & - & 6 & 272 & NS 97/95 & Serbia & 5 \\
\hline 60 & F5 5065-2 & Romania & 1 & 131 & NSP88 & Serbia & 5 & 202 & WWMCB 339 & - & 5 & 273 & NS 98/95 & Serbia & 6 \\
\hline 61 & BCD 1186/83 & Moldavia & 1 & 132 & Dina & Serbia & 5 & 203 & WWMCB 338 & - & 2 & 274 & NS $124 / 95$ & Serbia & 5 \\
\hline 62 & BCD 1286/83 & Moldavia & 5 & 133 & NSP199 & Serbia & 5 & 204 & Campodoro Cont. & Italy & 5 & 275 & Danica & Serbia & 5 \\
\hline 63 & BCD 1295/83 & Moldavia & 1 & 134 & NS135/90 & Serbia & 5 & 205 & CR-8 & - & 5 & 276 & Proteinka & Serbia & 5 \\
\hline 64 & BCD 1304/83 & Moldavia & 1 & 135 & NS10/94 & Serbia & 5 & 206 & CR-10 & - & 5 & 277 & NSP 51 & Serbia & 5 \\
\hline 65 & NSP187 & Serbia & 1 & 136 & NS39/93 & Serbia & 5 & 207 & 2004 & - & 5 & 278 & NSA 89-5126 & France & 5 \\
\hline 66 & L69/92 & Serbia & 1 & 137 & Jubilejnaja50 & Russia & 5 & 208 & 2005 & - & 5 & 279 & L 131/94 & Serbia & 5 \\
\hline 67 & Norin50 & Japan & 1 & 138 & Odeska 51 & Ukraine & 5 & 209 & 2017 & - & 5 & 280 & L 351/94 & Serbia & 5 \\
\hline 68 & Norin61 & Japan & 1 & 139 & Pavlovska 102 & Russia & 5 & 210 & 3015 & - & 5 & 281 & NS 50-14 & Serbia & 5 \\
\hline 69 & Peking 1-38 & China & 1 & 140 & Dnjestrovskaja 25 & Russia & 5 & 211 & 3017 & - & 5 & 282 & Mironovska 10 & Ukraine & 5 \\
\hline 70 & No4 & China & 2 & 141 & Elkohorn & - & 6 & 212 & 3020 & - & 5 & 283 & Raduša & Serbia & 6 \\
\hline 71 & Hang Chou & China & 1 & 142 & Atlas 66 & USA & 6 & 213 & 3002 & - & 5 & 284 & Chinese Spring & Control & \\
\hline
\end{tabular}

${ }^{(1)}$ GBR, Great Britain. ${ }^{(2)}$ (Maris Hobbit). ${ }^{(3)}$ var. duhamelianum. 
peduncle length, number of spikelets per spike, number of sterile spikelets per spike, spike index, spike weight, grain weight per spike, and grain number per spike.

Genomic DNA from all varieties (approximately ten plantlets per genotype) was isolated from fresh young leaves using the CTAB protocol described by Doyle \& Doyle (1990). Wheat genotype population was profiled with 30 microsatellite markers out of 41 initial markers, excluding 11 with non-specific PCR products. The sequences of SSR markers were taken from the GrainGenes database (GrainGenes, 2014) (Table 2). The additional variety Chinese Spring was used as a positive control. Microsatellites were positioned along almost all three genomes and located near previously detected important QTLs. PCR amplifications were carried out according to the protocols given by Röder et al. (1998). The reaction in $10 \mu \mathrm{L}$ volume contained $30 \mathrm{ng}$ of DNA template, $1 \mathrm{x}$ buffer solution, $2 \mathrm{mmol} \mathrm{L}^{-1}$ dNTPs, $1.5 \mathrm{mmol} \mathrm{L}^{-1} \mathrm{MgCl}_{2}, 10 \mathrm{pmol}$ of fluorescently labeled forward and unlabeled reverse primers, and 1 unit of Taq polymerase. PCR started with an initial denaturation at $94^{\circ} \mathrm{C}$ for $5 \mathrm{~min}$, followed by 40 cycles of $94^{\circ} \mathrm{C}$ for $30 \mathrm{~s}, 52-62^{\circ} \mathrm{C}$ for $45 \mathrm{~s}$, and $72^{\circ} \mathrm{C}$ for $45 \mathrm{~s}$. The final extension was $10 \mathrm{~min}$ at $72^{\circ} \mathrm{C}$. The PCR amplicons were separated by size using capillary electrophoresis on an ABI Prism 3130 genetic analyzer (Applied Biosystems, Foster City, CA, USA). The reaction volume of $10 \mu \mathrm{L}$ consisted of $2 \mu \mathrm{L}$ of mixed differently-labeled PCR products, $0.2 \mu \mathrm{L}$ of GeneScan 500 LIZ size standard (Applied Biosystems, Foster City, CA, USA), and $7.8 \mu \mathrm{L}$ of Hi-Di formamide. The dye-labeled products were identified by fluorescence detection, and microsatellite analysis was performed using the GeneMapper software, version 4.0 (Applied Biosystems, Foster City, CA, USA).

The parameters of genetic diversity were calculated with the PowerMarker software, version 3.25 (Liu $\&$ Muse, 2005). The population structure based on genetic data was estimated by the Bayesian algorithm implemented in the Structure software, version 2.3.4 (Pritchard et al., 2000). The hypothetical number of clusters was set ranging from 1 to 20 , whereas the length of the burn-in and the Markov chain Monte Carlo (MCMC) were determined at 100.000. The real number of subpopulations was obtained by comparing $\log$ probabilities of data $\operatorname{Pr}[\mathrm{X} \mid \mathrm{K}]$, and corrections were done according to Evanno et al. (2005). The selection of the most appropriate number of subgroups was a critical step for further association analysis. Determination of internal genetic structure was done by additional analysis through principal coordinate analysis (PCoA).

The marker-trait associations were analyzed in the Tassel software, version 2.1. (Bradbury et al., 2007) using two models: GLM and MLM (Yu et al., 2006). The $Q$ matrix for further association analysis was determined based on the average value of three iterations of $\log$ probability of data obtained by the Structure software (Pritchard et al., 2000). In order to define the level of genetic covariance between pairs of individuals, a kinship (K) analysis was carried out by molecular data, converting the distance matrix to a similarity matrix using the Tassel software (Bradbury et al., 2007). The magnitude of QTL effects was explained by the $\mathrm{R}^{2}$ parameter. The descriptive statistics of all phenotypic data was performed in the Statistica software, version 10 (Statsoft, Tulsa, OK, USA).

\section{Results and Discussion}

A total of 349 alleles was detected in 30 SSR loci, and the mean number of alleles per loci was 11.5 (Table 3 ). This result was higher than the diversity (7.2) found among USA wheat accessions (Chao et al., 2007). Chen et al. (2003) reported extremely low values of mean alleles per locus and other polymorphism parameters as a result of the genotype's specific region of origin, which led to a narrowing of genetic diversity. The sufficient genetic variation observed in the material evaluated in the present study was confirmed in other studies with the materials from the same core collection (Kobiljski et al., 2002). However, since the previous analysis was performed on only 96 genotypes, the mean average number of alleles per locus (7.96) was lower than in the present study. The average number of polymorphic information content (PIC) value was 0.688 , representing a highly significant level of genetic polymorphism. Considering the cosmopolitan origin of the studied varieties (Table 1), the breeding material indicates a broad genetic diversity that proved to be an excellent base for further research.

The population structure distributed genotypes into six subpopulations using log probability of data obtained by the Structure software (Figure 1), whereas the corrections of the number of clusters $(\Delta \mathrm{K})$ according to Evanno et al. (2005) indicated the distribution of genotypes into three existing subpopulations 
(Figure 2). Evanno's corrections generally predicted the existence of two or three subpopulations regardless of the number and diversity of the investigated materials (Vigouroux et al., 2008), which was confirmed in the present study. The classification of 283 genotypes was more effective in discriminating the genotypes toward log probability of data. The largest group (Q5) consisted of 114 genotypes, mainly originating from Serbia, whereas the smallest group (Q3) included 18 cultivars, mostly from the USA. The other subpopulations consisted of 37 genotypes (Q1), with diverse geographic origin; 45 genotypes (Q2),

Table 2. Microsatellite markers, sequences of forward and reverse primers, annealing temperature (Tm), repeated motif, and expected amplicons in the Chinese Spring variety of wheat (Triticum aestivum), used as a positive control.

\begin{tabular}{|c|c|c|c|c|c|}
\hline SSR markers & $\begin{array}{c}\text { Forward } \\
\text { primer }\end{array}$ & $\begin{array}{c}\text { Reverse } \\
\text { primer }\end{array}$ & $\begin{array}{l}\mathrm{Tm} \\
\left({ }^{\circ} \mathrm{C}\right)\end{array}$ & $\begin{array}{c}\text { Repeated } \\
\text { motif }\end{array}$ & $\begin{array}{c}\text { Expected } \\
\text { amplicons (bp) }\end{array}$ \\
\hline CFD65 (1A) & 5' AGA CGA TGA GAA GGA AGC CA 3' & 5' CCT CCC TTG TTT TTG GGA TT 3' & 62 & $(\mathrm{CT}) 32$ & 199 \\
\hline WMC333 (1A) & 5` TCA AGC ATA GGT GGC TTC GG 3’ & 5' ACA GCA GCC TTC AAG CGT TC 3' & 62 & (GT)15 & 174 \\
\hline CFA2086 (2A) & 5' TCT ACT TTC AGG GCA CCT CG 3' & 5' TCT CTC CAA ACC TCC CTG TAA 3' & 62 & (CA)21 & 220 \\
\hline WMC170 (2A) & $5^{`}$ ACA TCC ACG TTT ATG TTG TTG C 3’ & 5`TTG GTT GCT CAA CGT TTA CTT C 3` & 62 & (CA) 19 & 230 \\
\hline GWM294 (2A) & 5' GGA TTG GAG TTA AGA GAG AAC CG3' & 5' GCA GAG TGA TCA ATG CCA GA 3' & 62 & (GA)9TA(GA) 15 & - \\
\hline WMC407 (2A) & 5' GGT AAT TCT AGG CTG ACA TAT GCT C 3' & 5' CAT ATT TCC AAA TCC CCA ACT C 3, & 62 & (GA)16 & 135 \\
\hline CFD71 (4A) & 5' CAA TAA GTA GGC CGG GAC AA 3' & 5' TGT GCC AGT TGA GTT TGC TC 3' & 62 & $(\mathrm{CA}) 10(\mathrm{GA}) 30$ & 216 \\
\hline GWM160 (4A) & 5' TTC AAT TCA GTC TTG GCT TGG 3' & 5' CTG CAG GAA AAA AAG TAC ACC C 3' & 62 & $(\mathrm{GA}) 21$ & - \\
\hline CFA2114 (6A) & 5' ATT GGA AGG CCA CGA TAC AC 3' & 5' CCC GTC GGG TTT TAT CTA GC 3' & 62 & (CA)32 & 209 \\
\hline WMC333 (6A) & 5` TCA AGC ATA GGT GGC TTC GG 3’' & 5' ACA GCA GCC TTC AAG CGT TC 3' & 62 & (GT)15 & 174 \\
\hline CFA2257 (7A) & 5' GAT ACA ATA GGT GCC TCC GC 3' & 5' CCA TTA TGT AAA TGC TTC TGT TTG A 3' & 60 & (TG)28 & 167 \\
\hline WMC83 (7A) & 5`TGG AGG AAA CAC AAT GGA TGC C 3’ & $5^{`}$ GAG TAT CGC CGA CGAAAG GGA A $3 `$ & 62 & (GT)28 & 160 \\
\hline GWM11 (1B) & $5^{`}$ GGA TAG TCA GAC AAT TCT TGT G 3' & $5^{`}$ GTG AAT TGT GTC TTG TAT GCT TCC $3{ }^{`}$ & 52 & (TA)6CATA(CA)19(TA)6 & 196 \\
\hline WMC44 (1B) & 5` GGT CTT CTG GGC TTT GAT CCT G 3’' & $5^{`}$ TGT TGC TAG GGA CCC GTA GTG G 3` & 52 & (GT)35 & 242 \\
\hline GWM148 (2B) & 5' GTG AGG CAG CAA GAG AGA AA 3' & 5' CAA AGC TTG ACT CAG ACC AAA 3' & 62 & (CA)22 & - \\
\hline BARC101 (2B) & 5' GCT CCT CTC ACG ATC ACG CAA AG 3' & 5' GCG AGT CGA TCA CAC TAT GAG CCA ATG 3' & 55 & $(\mathrm{TAA}) 9$ & - \\
\hline WMC154 (2B) & $5^{`}$ ATG CTC GTC AGT GTC ATG TTT G 3’ & 5' AAA CGG AAC CTA CCT CAC TCT T 3' & 62 & $(\mathrm{GT}) 34$ & 147 \\
\hline GWM181 (3B) & 5' TCA TTG GTA ATG AGG AGA GA 3' & 5' GAA CCA TTC ATG TGC ATG TC 3' & 52 & (GA)28 & - \\
\hline GWM368 (4B) & 5' CCA TTT CAC CTA ATG CCT GC 3' & 5' AAT AAA ACC ATG AGC TCA CTT GC 3' & 62 & (AT) 25 & - \\
\hline WMC28 (5B) & $5^{`}$ ATC ACG CAT GTC TGC TAT GTA T 3’' & $5 `$ ATT AGA CCA TGA AGA CGT GTA T 3’ & 51 & $(\mathrm{CA})(\mathrm{T})(\mathrm{CA})(\mathrm{GT}) 29$ & 188 \\
\hline GWM271 (5B) & 5' CAA GAT CGT GGA GCC AGC 3' & 5' AGC TGC TAG CTT TTG GGA CA 3' & 62 & (CT)4imp(GA) 10 & - \\
\hline GWM219 (6B) & 5' GAT GAG CGA CAC CTA GCC TC 3' & 5' GGG GTC CGA GTC CAC AAC 3' & 60 & (GA)35imp & - \\
\hline WMC166 (7B) & 5’ ATA AAG CTG TCT CTT TAG TTC G 3' & $5 `$ GTT TTA ACA CAT ATG CAT ACC T 3’ & 55 & $(\mathrm{GA}) 8(\mathrm{GT}) 8(\mathrm{GT}) 8$ & 305 \\
\hline WMC216 (1D) & $5^{`}$ ACG TAT CCA GAC ACT GTG GTA A 3' & $5^{`}$ TAA TGG TGG ATC CAT GAT AGC C $3 `$ & 55 & (GT)22 & 123 \\
\hline WMC167 (2D) & $5^{`}$ AGT GGT AAT GAG GTG AAA GAA G 3’ & 5` TCG GTC GTA TAT GCA TGT AAA G 3` & 52 & $(\mathrm{CA}) 22(\mathrm{CA}) 8(\mathrm{CA}) 8$ & 185 \\
\hline WMC18 (2D) & 5’ CTG GGG CTT GGA TCA CGT CAT T 3’' & 5` AGC CAT GGA CAT GGT GTC CTT C 3' & 62 & $(\mathrm{CA})(\mathrm{CT})$ & 230 \\
\hline GWM157 (2D) & 5' GTC GTC GCG GTA AGC TTG 3' & 5' GAG TGA ACA CAC GAG GCT TG 3' & 62 & $(\mathrm{CT}) 14$ & - \\
\hline WMC144 (2D) & $5{ }^{\prime}$ GGA CAC CAA TCC AAC ATG AAC A 3' & 5'AAG GAT AGT TGG GTG GTG CTG A 3' & 62 & (CA) 14 & 143 \\
\hline GWM292 (5D) & 5' TCA CCG TGG TCA CCG AC 3' & 5' CCA CCG AGC CGA TAA TGT AC 3' & 62 & (CT)38 & - \\
\hline PSP3200 (6D) & 5’ GTT CTG AAG ACA TTA CGG ATG 3' & 5’ GAG AAT AGC TGG TTT TGT GG 3’ & 62 & $(\mathrm{AAG}) 16$ & 170 \\
\hline \multicolumn{6}{|c|}{ Markers with non-specific products } \\
\hline BARC124 (2A, 2B) & 5' TGC ACC CCT TCC AAA TCT 3' & 5' TGC GAG TCG TGT GGT TGT 3' & 52 & (CT) 19 & - \\
\hline CFD38 (6D) & 5' TGG CCA TTC GAT ATT CAA AA 3' & 5' GTG AGT TGA GGC GCA TGA TA 3' & 60 & $(\mathrm{GA}) 32$ & 215 \\
\hline WMC657 (4B) & 5' CGG GCT GCG GGG GTA T 3' & 5' CGG TTG GGT CAT TTG TCT CA 3' & 61 & - & 119 \\
\hline WMC664 (3A) & 5' GGG CCA ACA AAT CCAAT 3' & 5' TCT ACT TCC TTC ATC CAC TCC 3' & 61 & - & 157 \\
\hline WMC625 (3A, 3B) & 5' CAC AGA CCT CAA CCT CTT CTT 3’ & 5' AGT ACT GTT CAC AGC AGA CGA 3' & 61 & - & 113 \\
\hline WMC413 (4B) & $5{ }^{\circ}$ CAC TGG AAA CAT CTC TTC AAC T $3{ }^{\prime}$ & 5' ACA GGA AAG GAT GAT GTT CTC T 3' & 51 & $(\mathrm{GT}) 9$ & 162 \\
\hline GWM257 (2B) & 5'AGA GTG CAT GGT GGG ACG 3' & 5' CCA AGA CGA TGC TGA AGT CA 3' & 60 & (GT)30 & - \\
\hline WMC765 (5D) & 5` GGG ATC AGA CTG GGA CTG GAG 3' & 5' GGG TTG GCT TGG CAG AGA A 3' & 61 & - & 167 \\
\hline CFD26 (5D) & 5' TCA AGA TCG TGC CAA ATC AA 3' & 5'ACT CCA AGC TGA GCA CGT TT 3' & 60 & (GAGAA)2(GA)37 & 271 \\
\hline WMC245 (2D, 2B) & 5` GCT CAG ATC ATC CAC CAA CTT C 3' & 5' AGA TGC TCT GGG AGA GTC CTT A 3' & 61 & - & 129,150 \\
\hline GWM494 (4A) & 5' ATT GAA CAG GAA GAC ATC AGGG 3' & 5' TTC CTG GAG CTG TCT GGC 3' & - & (CA)13 & - \\
\hline
\end{tabular}


mostly from England and France; 30 genotypes (Q4), from Croatia; and 40 genotypes (Q6), from the USA.
The distribution could be explained partly by geographical origin and partly by pedigree data.

Table 3. Basic parameters of genetic diversity in wheat (Triticum aestivum).

\begin{tabular}{|c|c|c|c|c|c|c|c|}
\hline SSR markers & Chromosome & $\begin{array}{c}\text { Allelic No/ } \\
\text { locus }\end{array}$ & $\begin{array}{c}\text { Most frequent } \\
\text { allele }\end{array}$ & PIC & $\begin{array}{c}\text { Gene } \\
\text { diversity }\end{array}$ & $\begin{array}{c}\text { Observed } \\
\text { heterozygosity }\end{array}$ & $\begin{array}{c}\text { Allele size } \\
\text { (bp) }\end{array}$ \\
\hline WMC28 & $5 B$ & 10 & 0.356 & 0.706 & 0.746 & 0 & 177-203, null \\
\hline CFD65 & $1 \mathrm{~A}$ & 10 & 0.271 & 0.739 & 0.777 & 0.004 & 180-202, null \\
\hline GWM292 & $5 \mathrm{D}$ & 14 & 0.342 & 0.777 & 0.802 & 0 & 196-222, null \\
\hline WMC167 & $2 \mathrm{D}$ & 13 & 0.649 & 0.516 & 0.545 & 0.028 & $141-191$ \\
\hline GWM271 & $5 B$ & 7 & 0.528 & 0.626 & 0.663 & 0.004 & 142-166, null \\
\hline CFA2257 & 7A & 6 & 0.570 & 0.426 & 0.525 & 0.021 & $123-141$ \\
\hline WMC83 & $7 \mathrm{~A}$ & 9 & 0.289 & 0.799 & 0.823 & 0 & 151-165, null \\
\hline GWM148 & $2 \mathrm{~B}$ & 11 & 0.269 & 0.785 & 0.812 & 0.011 & 140-168, null \\
\hline PSP3200 & $6 \mathrm{D}$ & 7 & 0.391 & 0.701 & 0.742 & 0.018 & 159-177, null \\
\hline GWM181 & $3 \mathrm{~B}$ & 19 & 0.232 & 0.827 & 0.846 & 0.176 & $117-157$, null \\
\hline CFA2086 & $2 \mathrm{~A}$ & 24 & 0.190 & 0.896 & 0.905 & 0.004 & 207-275, null \\
\hline WMC170 & $2 \mathrm{~A}$ & 10 & 0.366 & 0.701 & 0.741 & 0.218 & 182-226, null \\
\hline GWM11 & $1 \mathrm{~B}$ & 11 & 0.300 & 0.802 & 0.825 & 0 & 184-206, null \\
\hline WMC44 & $1 \mathrm{~B}$ & 21 & 0.379 & 0.801 & 0.816 & 0.014 & 204-266, null \\
\hline CFA2114 & $6 \mathrm{~A}$ & 12 & 0.236 & 0.837 & 0.855 & 0 & 199-221, null \\
\hline BARC101 & $2 \mathrm{~B}$ & 10 & 0.535 & 0.619 & 0.655 & 0.011 & 102-130, null \\
\hline WMC216 & $1 \mathrm{D}$ & 12 & 0.794 & 0.354 & 0.364 & 0.004 & 106-132, null \\
\hline GWM294 & $2 \mathrm{~A}$ & 19 & 0.229 & 0.858 & 0.871 & 0.004 & $65-111$ \\
\hline WMC166 & $7 \mathrm{~B}$ & 4 & 0.634 & 0.513 & 0.553 & 0 & 306-310, null \\
\hline WMC18 & $2 \mathrm{D}$ & 14 & 0.284 & 0.830 & 0.847 & 0.004 & 218-248, null \\
\hline CFD71 & $4 \mathrm{~A}$ & 16 & 0.180 & 0.876 & 0.888 & 0.007 & 169-207, null \\
\hline GWM157 & $2 \mathrm{D}$ & 7 & 0.826 & 0.297 & 0.309 & 0.014 & $77-107$ \\
\hline WMC144 & $2 \mathrm{D}$ & 6 & 0.582 & 0.425 & 0.522 & 0 & $137-157$ \\
\hline WMC407 & $2 \mathrm{~A}$ & 11 & 0.379 & 0.738 & 0.769 & 0.004 & $121-153$ \\
\hline WMC333-1A & $1 \mathrm{~A}$ & 3 & 0.512 & 0.400 & 0.517 & 0 & $157-161$ \\
\hline WMC333-6A & $6 \mathrm{~A}$ & 6 & 0.466 & 0.552 & 0.626 & 0 & $161-183$ \\
\hline GWM160 & $4 \mathrm{~A}$ & 8 & 0.248 & 0.785 & 0.813 & 0.004 & 166-186, null \\
\hline WMC154 & $2 \mathrm{~B}$ & 15 & 0.220 & 0.818 & 0.840 & 0.004 & $117-161$ \\
\hline GWM219 & $6 \mathrm{~B}$ & 15 & 0.166 & 0.875 & 0.888 & 0 & 163-195, null \\
\hline GWM368 & 4B & 19 & 0.387 & 0.766 & 0.789 & 0 & 202-258, null \\
\hline Total/average & & $349 / 11.5$ & 0.394 & 0.688 & 0.722 & 0.020 & \\
\hline
\end{tabular}

PIC, polymorphic information content.

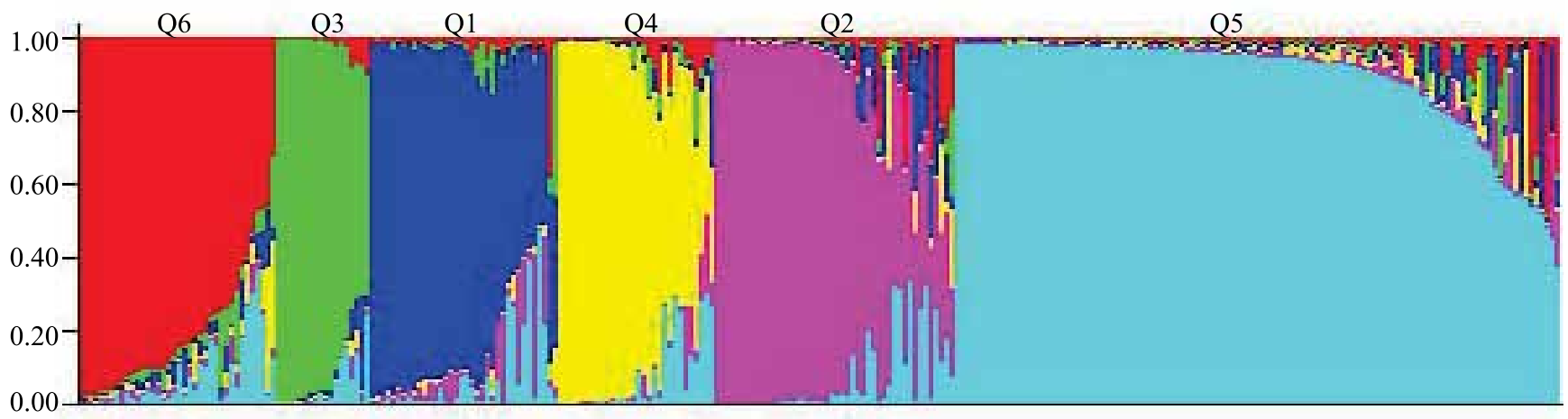

Figure 1. Population structure of 283 wheat (Triticum aestivum) genotypes estimated using the model-based Bayesian algorithm implemented in the Structure software (Pritchard et al., 2000) performed with 30 microsatellite loci. Q1 to Q6, genotype clusters on the Q matrix. 


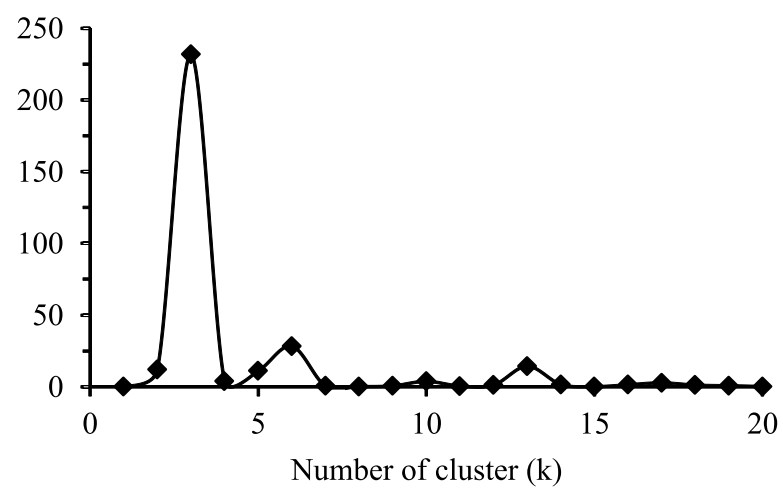

Figure 2. Correction of number of clusters $(\Delta \mathrm{K})$ according to Evanno et al. (2005) for the different Bayesian clustering analyses implemented by the Structure software (Pritchard et al., 2000).
Likewise, strict distribution according to origin is difficult because of the use of breeding and elite lines through and by different breeding centers. Even the distribution of genotypes originating from the same regions points to a similar selective pressure in wheat breeding during domestication and the subsequent breeding process (Laido et al., 2013). Moreover, internal genetic structure using PCoA separated the largest subpopulation (Q5) and group (Q2) mostly consistent with grouping by the Structure software (Figure 3). In addition, the groups from Croatia (Q4) and from the USA (Q3) took a particular position in the coordinate system, whereas the remaining two clusters (Q1 and Q6) showed dispersed distribution in the coordinate system. However, certain overlapping

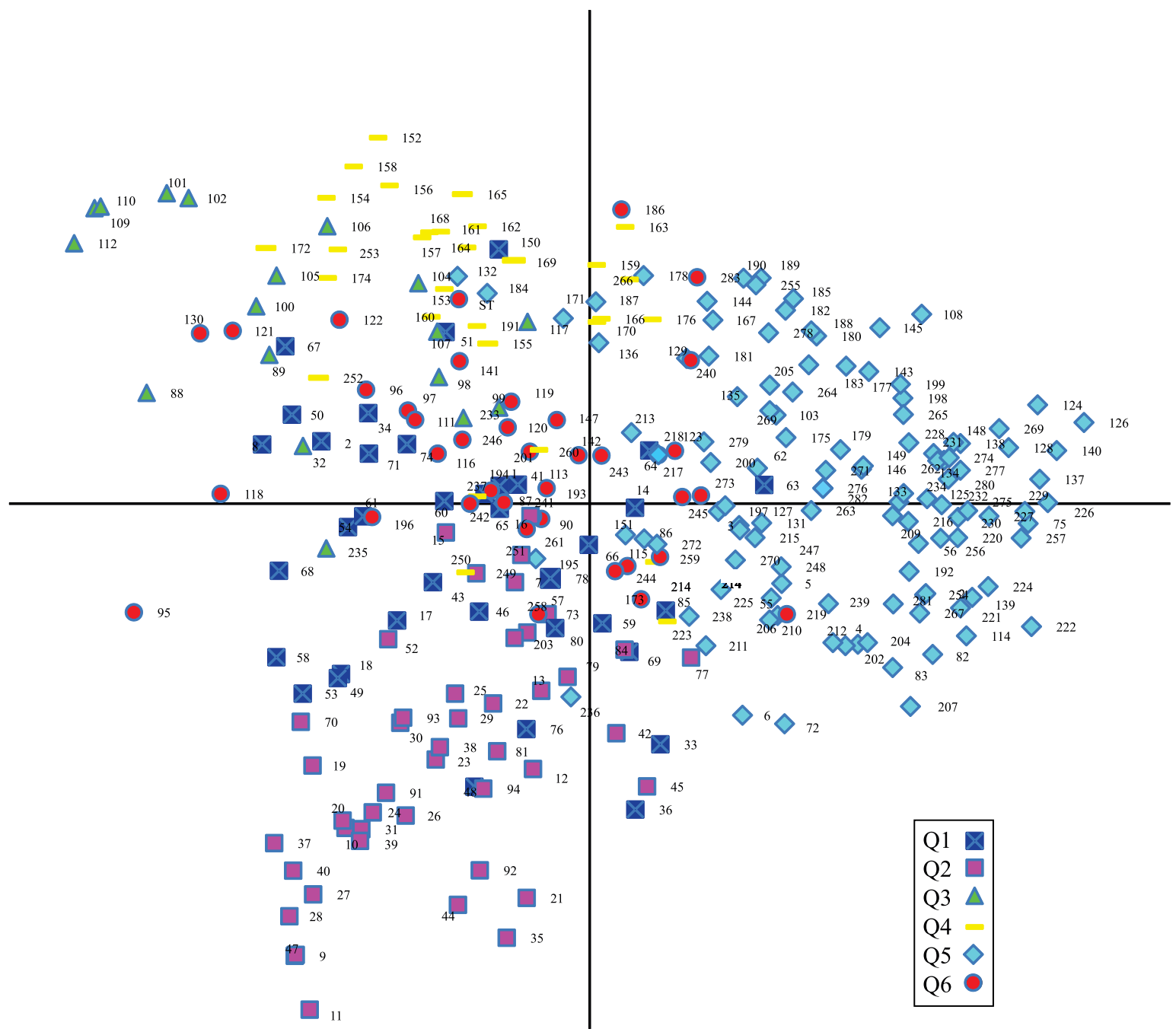

Figure 3. Principal coordinate analysis of the 283 wheat (Triticum aestivum) varieties. Each mark represents a sample obtained by the Structure software (Pritchard et al., 2000). Q1 to Q6, genotype clusters on the Q matrix. 
within some subpopulations could be a result of the frequent use of certain varieties as parents, as well as of the inclusion of a great number of genotypes into the analysis. Population structure determined by model-based clustering in the Structure software was the most appropriate tool for determining genetic structure and a key component for further association studies (Yu et al., 2006).

The total number of detected marker-trait associations in the five evaluation years was of 192 using the GLM method, but decreased to 76 for all analyzed traits and years using the MLM approach (Table 4). The advantage of the MLM approach is the detection of more real loci associated with agronomic traits, without false positive associations (Zhang et al., 2013). Neumann et al. (2011) suggested the usefulness of both models because a great number of associations could be neglected using only the MLM, resulting in many MTAs that might not be recognized as potential

Table 4. Total number of marker-trait associations $(\mathrm{p} \leq 0.01)$ detected with the general linear model (GLM) and the mixed linear model (MLM) methods in three evaluation years.

\begin{tabular}{lcccc}
\hline Traits & \multicolumn{2}{c}{$\begin{array}{c}\text { Significant GLM Significant MLM } \\
\text { associations }\end{array}$} & $\begin{array}{c}\text { Unique } \\
\text { associations }\end{array}$ & $\begin{array}{c}\text { Unique } \\
\text { GLM }\end{array}$ \\
\hline Spikelets per spike & 42 & 22 & 34 & 0 \\
SSS $^{(1)}$ & 46 & 18 & 30 & 0 \\
Grains per spike & 30 & 16 & 15 & 1 \\
Spike length & 29 & 7 & 28 & 0 \\
Spike weight & 12 & 4 & 17 & 0 \\
GWS & $(2)$ & 4 & 12 & 0 \\
Spike index & 12 & 3 & 24 & 0 \\
Peduncle length & 9 & 2 & 7 & 1 \\
\hline Total & 192 & 76 & 167 & 2 \\
\hline${ }^{(1)}$ SSS, sterile spikelets per spike. ${ }^{(2)}$ GWS, grain weight per spike.
\end{tabular}

loci. This statement is in accordance with $\mathrm{Yu}$ et al. (2009), who proposed that new loci detected by GLM are also useful and should be additionally validated to avoid false-positive associations. Furthermore, the differences detected by these two models could be trait-dependent (Neumann et al., 2011).

It is important to highlight that only the stable associations detected in more than three evaluation years, at $1 \%$ probability, using the GLM and MLM approaches, were reported (Table 5). Four closely located markers (wmc18, wmc167, wmc144, and gwm157) on chromosome 2D were significant for the detection of QTLs for number of spikelets per spike, number of sterile spikelets per spike, and grain number per spike. This observation agrees with the results of high partial correlations obtained for these traits (Table 6). Besides being a carrier of three key genes for height reduction $(R h t 8)$, photoperiod ( $P p d 1)$, and yellow rust $(\operatorname{Yr} 16)$, which are essential for adaptation, chromosome 2D contained most markers associated with the agronomically important traits.

The proximity region of the Ppd-1 gene, near gwm 484 , was responsible for the expression of many yield components and spike morphology, showing its high value for wheat improvement (Dodig et al., 2012). On the integrated genetic map of this chromosome created with scaffolds and markers in Aegilops tauschii, Jia et al. (2013) identified 33 QTLs or genes. One of them was the QTL for test weight near marker $w m c 167$, which was significant for spike-related traits in the present study. Marker wmc144 showed the highest effect on phenotypic variation of spikelets per spike with mean value of $40.7 \%$. QTLs for grain number per spike and spike length were found in association with marker gwm 294 , derived by Yao et al. (2009), located on the long arms of chromosome 2A.

Table 5. Markers associated $(\mathrm{p} \leq 0.01)$ with spike-related traits in more than three evaluation years using the mixed linear model method, and the mean value of phenotypic variation $(\%)$.

\begin{tabular}{|c|c|c|c|c|c|c|c|c|c|}
\hline $\begin{array}{l}\text { Traits/ } \\
\text { markers }\end{array}$ & $\begin{array}{c}\text { GWM157 } \\
\text { 2D }\end{array}$ & $\begin{array}{c}\text { WMC } 144 \\
2 \mathrm{D}\end{array}$ & $\begin{array}{l}\text { WMC167 } \\
2 \mathrm{D}\end{array}$ & $\begin{array}{c}\text { WMC18 } \\
\text { 2D }\end{array}$ & $\begin{array}{c}\text { GWM11 } \\
\text { 1B }\end{array}$ & $\begin{array}{c}\text { BARC101 } \\
2 \mathrm{~B}\end{array}$ & $\begin{array}{c}\text { GWM294 } \\
\text { 2A }\end{array}$ & $\begin{array}{c}\text { CFA2086 } \\
\text { 2A }\end{array}$ & $\begin{array}{c}\text { WMC333-2 } \\
6 \mathrm{~A}\end{array}$ \\
\hline Spikelets per spike & 12.2 & 40.7 & 26.6 & 13.7 & ns & ns & ns & ns & ns \\
\hline Sterile spikelets per spike & 9.7 & 8.6 & 22.2 & 14.6 & 10.7 & ns & ns & ns & ns \\
\hline Grains per spike & 7.6 & ns & 11 & 8.5 & ns & 8.2 & 9.8 & ns & ns \\
\hline Spike index & $\mathrm{ns}$ & ns & $\mathrm{ns}$ & $\mathrm{ns}$ & $\mathrm{ns}$ & ns & ns & 14.3 & $\mathrm{~ns}$ \\
\hline Peduncle length $^{(1)}$ & ns & ns & ns & ns & ns & ns & 12 & 11.1 & ns \\
\hline Spike length & 7.6 & ns & ns & ns & ns & ns & 11 & 14.6 & 4.7 \\
\hline
\end{tabular}

${ }^{(1)}$ Measured only in three years, and significant marker-trait associations in more than two years. ${ }^{\text {ns}}$ Nonsignificant. 
Table 6. Partial correlations of significant phenotypic traits with mean values for each genotype and coefficient of variation (CV) for each trait.

\begin{tabular}{|c|c|c|c|c|c|c|c|c|c|}
\hline Traits & $\begin{array}{l}\text { Spikelets per } \\
\text { spike }\end{array}$ & $\begin{array}{l}\text { Sterile spikelets } \\
\text { per spike }\end{array}$ & $\begin{array}{c}\text { Grains per } \\
\text { spike }\end{array}$ & $\begin{array}{l}\text { Spike } \\
\text { length }\end{array}$ & $\begin{array}{l}\text { Spike } \\
\text { index }\end{array}$ & $\begin{array}{c}\text { Spike } \\
\text { weight }\end{array}$ & $\begin{array}{l}\text { Grain weight } \\
\text { per spike }\end{array}$ & $\begin{array}{c}\text { Peduncle } \\
\text { length }\end{array}$ & $\begin{array}{l}\mathrm{CV} \\
(\%) \\
\end{array}$ \\
\hline Spikelets per spike & 1 & - & - & - & - & - & - & - & 33.26 \\
\hline Sterile spikelets per spike & $0.796 * *$ & 1 & - & - & - & - & - & - & 80.96 \\
\hline Grains per spike & $0.667^{* *}$ & $0.396^{* *}$ & 1 & - & - & - & - & - & 22.53 \\
\hline Spike length & $0.444 * *$ & $0.410 * *$ & $0.432 * *$ & 1 & - & - & - & - & 17.40 \\
\hline Spike index & $-0.330 * *$ & $-0.340 * *$ & $-0.159 * *$ & $-0.319 * *$ & 1 & - & - & - & 6.60 \\
\hline Spike weight & $0.409 * *$ & $0.181 * *$ & $0.742 * *$ & $0.399 * *$ & $0.115^{* *}$ & 1 & - & - & 20.94 \\
\hline Grain weight per spike & $0.256^{* *}$ & ns & $0.629^{* *}$ & $0.269 * *$ & $0.389^{* *}$ & $0.953^{* *}$ & 1 & - & 22.00 \\
\hline Peduncle length & $0.155^{* *}$ & $0.203 * *$ & ns & $0.278 * *$ & $0.085^{*}$ & $0.076^{*}$ & $0.076^{*}$ & 1 & 21.12 \\
\hline
\end{tabular}

In the present study, this marker showed similar effects on the phenotypic variation of these traits (13 and 5\%, respectively) (Table 5). Also, two markers (gwm 294 and $c f a 2086$ ) on chromosome $2 \mathrm{~A}$ were associated with peduncle length apart from the previously detected QTL for this trait on chromosome 6A (Neumann et al., 2011). This trait has attracted great interest in recent studies due to its importance in avoiding ear diseases. Grain number per spike is one of the most important yield components of wheat (Ma et al., 2007), which was associated with the largest number of markers evaluated, i.e., five (Table 5). The specific marker for grain number was barc101 (2BL), which has not been previously associated with this trait, indicating the presence of a new QTL. The presence of QTLs near marker gwm 11 for a large number of agronomic and adaptive traits has been proven by Wang et al. (2009), whereas, in the present study, the only association of this marker was found with sterile spikelets per spike. Only a limited number of QTL studies for sterile spikelet number per spike have been documented (Ma et al., 2007). The coefficient of variation for sterile spikelets per spike obtained by descriptive statistics was extremely high (Table 6), probably due to the selection of a relatively small number of varieties with branched architecture of wheat spikes. Grain weight per spike and spike weight were the only traits with absence of stable associations in more evaluation years. Using the collection of genotypes with a high level of polymorphism for association analysis and finding stable QTLs over a course of multiple years could be useful for the breeding process (Maccaferri et al., 2008). A potential new flowering-time gene on chromosome 6D ( $p s p 3200)$ was detected in similar material from the same core collection under contrasting water regimes
(Dodig et al., 2012). However, this region has not shown importance for spike characteristics considering field conditions. The unique association between marker $w m c 333$ on chromosome $6 \mathrm{~A}$ and spike length detected in the present study could indicate the presence of new potential QTL with minor effect.

\section{Conclusions}

1. The evaluated collection of wheat (Triticum aestivum) genotypes shows genetic diversity, and population structure is an important tool for association analysis.

2. A significant number of associations is stable for six spike-related traits.

3. The statistical models evaluated increase the accuracy and power of the association analysis.

4. The new chromosome regions identified as responsible for spike-related traits are useful for wheat breeding programs.

\section{Acknowledgments}

To the Ministry of Education, Science and Technological development of Serbia (Project number TR31066), for support.

\section{References}

BRADBURY, P.J.; ZHANG, Z.; KROON, D.E.; CASSTEVENS, T.M.; RAMDOSS, Y.; BUCKLER, E.S. TASSEL: software for association mapping of complex traits in diverse samples. Bioinformatics, v.23, p.2633-2635, 2007. DOI: 10.1093/ bioinformatics/btm308.

BRESEGHELLO, F.; SORRELLS, M.E. Association analysis as a strategy for improvement of quantitative traits in plants. 
Crop Science, v.46, p.1323-1330, 2006. DOI: 10.2135/ cropsci2005.09-0305.

CHAO, S.M.; ZHANG, W.J.; DUBCOVSKY, J.; SORRELS, M. Evaluation of genetic diversity and genome-wide linkage disequilibrium among U.S. wheat (Triticum aestivum L.) germplasm representing different market classes. Crop Science, v.47, p.1018-1030, 2007. DOI: 10.2135/cropsci2006.06.0434.

CHEN, X.M.; HE, Z.H.; SHI, J.R.; XIA, L.Q.; WARD, R.; ZHOU, Y.; JIANG, G.L. Genetic diversity of high quality winter wheat varieties (lines) based on SSR markers. Acta Agronomica Sinica, v.29, p.13-19, 2003.

CUTHBERT, J.L.; SOMERS, D.J.; BRULE-BABEL, A.L.; BROWN, P.D.; CROW, G.H. Molecular mapping of quantitative trait loci for yield and yield components in spring wheat (Triticum aestivum L.). Theoretical and Applied Genetics, v.117, p.595-608, 2008. DOI: $10.1007 / \mathrm{s} 00122-008-0804-5$.

DODIG, D.; ZORIĆ, M.; KOBILJSKI, B.; SAVIĆ, J.; KANDIĆ, V.; QUARRIE, S.; BARNES, J. Genetic and association mapping study of wheat agronomic traits under contrasting water regimes. International Journal of Molecular Sciences, v.13, p.6167-6188, 2012. DOI: 10.3390/ijms 13056167.

DOYLE, J.J.; DOYLE, J.L. Isolation of plant DNA from fresh tissue. Focus, v.12, p.13-15, 1990.

EVANNO, G.; REGNAUT, S.; GOUDET, J. Detecting the number of clusters of individuals using the software STRUCTURE: a simulation study. Molecular Ecology, v.14, p.2611-2620, 2005. DOI: 10.1111/j.1365-294X.2005.02553.x.

GRAINGENES: a database for Triticeae and Avena. Available at: $<$ http://wheat.pw.usda.gov/GG2/index.shtml $>$. Accessed on: 10 Sept. 2014.

HASENEYER, G.; STRACKE, S.; PIEPHO, H.-P.; SAUER, S.; GEIGER, H.H.; GRANER, A. DNA polymorphisms and haplotype patterns of transcription factors involved in barley endosperm development are associated with key agronomic traits. BMC Plant Biology, v.10, p.1-11, 2010. DOI: 10.1186/1471-2229-10-5.

JIA, J.; ZHAO, S.; KONG, X.; LI, Y.; ZHAO, G.; HE, W.; APPELS, R.; PFEIFER, M.; TAO, Y.; ZHANG, X.; JING, R.; ZHANG, C.; MA, Y.; GAO, L.; GAO, C.; SPANNAGL, M.; MAYER, K.F.X.; LI, D.; PAN, S.; ZHENG, F.; HU, Q.; XIA, X.; LI, J.; LIANG, Q.; CHEN, J.; WICKER, T.; GOU, C.; KUANG, H.; HE, G.; LUO, Y.; KELLER, B.; XIA, Q.; LU, P.; WANG, J.; ZOU, H.; ZHANG, R.; XU, J.; GAO, J.; MIDDLETON, C.; QUAN, Z.; LIU, G.; WANG, J.; INTERNATIONAL WHEAT GENOME SEQUENCING CONSORTIUM; YANG, H.; LIU, X.; HE, Z.; MAO, L.; WANG, J. Aegilops tauschii draft genome sequence reveals a gene repertoire for wheat adaptation. Nature, v.496, p.91-95, 2013. DOI: 10.1038/ nature 12028.

KOBILJSKI, B.; QUARRIE, S.; DENČIĆ, S.; KIRBY, J.; IVEGES, M. Genetic diversity of the Novi Sad Wheat Core Collection revealed by microsatellites. Cellular and Molecular Biology Letters, v.7, p.685-694, 2002. DOI: 10.1038/nature12028.

LAIDO, G.; MANGINI, G.; TARANTO, F.; GADALETA, A.; BLANCO, A.; CATTIVELLI, L.; MARONE, D.; MASTRANGELO, A.M.; PAPA, R.; DE VITA, P. Genetic diversity and population structure of tetraploid wheats (Triticum turgidum L.) estimated by SSR, DarT and pedigree data. PloS ONE, v.8, e6720, 2013. DOI: 10.13717/journal.pone.0067280.

LANDJEVA, S.; KORZUN, V.; BÖRNER, A. Molecular markers: actual and potential contributions to wheat genome characterization and breeding. Euphytica, v.156, p.271-296, 2007. DOI: 10.1007/ s10681-007-9371-0.

LIU, K.J.; MUSE, S.V. PowerMarker: an integrated analysis environment for genetic marker analysis. Bioinformatics, v.21, p.2128-2129, 2005. DOI: 10.1093/bioinformatics/bti282.

LIU, L.; WANG, L.; YAO, J.; ZHENG, Y.; ZHAO, C. Association mapping of six agronomic traits on chromosome $4 \mathrm{~A}$ of wheat (Triticum aestivum L.). Molecular Plant Breeding, v.1, p.1-10, 2010.

MA, Z.; ZHAO, D.; ZHANG, C.; ZHANG, Z.; XUE, S.; LIN, F.; KONG, Z.; TIAN D.; LUO Q. Molecular genetic analysis of spike-related traits in wheat using RIL and immortalized F2 populations. Molecular Genetics and Genomics, v.277, p.31-42, 2007. DOI: 10.1007/s00438-006-0166-0.

MACCAFERRI, M.; SANGUINETI, M.C.; CORNETI, S.; ORTEGA, J.L.A.; SALEM, M.B.; BORT, J.; DEAMBROGIO, E.; GARCIA DEL MORAL, L.F.; DEMONTIS, A.; EL-AHMED, A.; MAALOUF, F.; MACHLAB, H.; MARTOS, V.; MORAGUES, M.; MOTAWAJ, J.; NACHIT, M.; NSERALLAH, N.; OUABBOU, H.; ROYO, C.; SLAMA, A.; TUBEROSA, R. Quantitative trait loci for grain yield and adaptation of durum wheat (Triticum durum Desf.) across a wide range of water availability. Genetics, v.178, p.489-511, 2008. DOI: 10.1534/genetics.107.077297.

MALEŠEVIĆ, M.; STARČEVIĆ, S.; MILOŠEV, D. Terms of cultivation and production technology of grain crops. In: FURMAN, T. (Ed.). The mechanized production of small grains. Novi Sad: Institute of Agriculture Technique, 1994. p.1-17.

NEUMANN, K.; KOBILJSKI, B.; DENČIĆ, S.; VARSHNEY, R.K.; BÖRNER, A. Genome-wide association mapping: a case study in bread wheat (Triticum aestivum L.). Molecular Breeding, v.27, p.37-58, 2011. DOI: 10.1007/s11032-010-9411-7.

PRITCHARD, J.K.; STEPHENS, M.; DONNELLY, P. Inference of population structure using multilocus genotype data. Genetics, v.155, p.945-959, 2000.

REHMAN ARIF, M.A.; NAGEL, M.; NEUMANN, K.; KOBILJSKI, B.; LOHWASSER, U. BÖRNER, A. Genetic studies of seed longevity in hexaploid wheat using segregation and association mapping approaches. Euphytica, v.186, p.1-13, 2012. DOI: $10.1007 / \mathrm{s} 10681-011-0471-5$.

REIF, J.C.; GOWDA, M.; MAURER, H.P.; LONGIN, C.F.H.; KORZUN, V.; EBMEYER, E.; BOTHE, R.; PIETSCH, C.; WÜRSCHUM, T. Association mapping for quality traits in soft winter wheat. Theoretical and Applied Genetics, v.122, p.961-970, 2011. DOI: 10.1007/s00122-010-1502-7.

RÖDER, M.S.; KORZUN, V.; WENDEHAKE, K.; PLASCHKE, J.; TIXIER, M-H.; LEROZ, P.; GANAL, M.W. A microsatellite map of wheat. Genetics, v.194, p.2007-2023, 1998.

VIGOUROUX, Y.; GLAUBITZ, J.C.; MATSUOKA, Y.; GOODMAN, M.M.; SANCHEZ, J.G.; DOEBLEY, J. Population structure and genetic diversity of new world maize races assessed 
by DNA microsatellites. American Journal of Botany, v.95, p.1240-1253, 2008. DOI: 10.3732/ajb.0800097.

WANG, M.; JIANG, N.; JIA, T.; LEACH, L.; COCKRAM, J.; WAUGH, R.; RAMSAY, L.; THOMAS, B.; LUO, Z. Genome-wide association mapping of agronomic and morphologic traits in highly structured populations of barley cultivars. Theoretical and Applied Genetics, v.124, p.233-246, 2012. DOI: 10.1007/s00122011-1697-2.

WANG, R.X.; HAI, L.; ZHANG, X.Y.; YOU, G.X.; YAN, C.S.; XIAO, S.H. QTL mapping for grain filling rate and yield-related traits in RILs of the Chinese winter wheat population Heshangmai x Yu8679. Theoretical and Applied Genetics, v.118, p.313-325, 2009. DOI: 10.1007/s00122-008-0901-5.

YAO, J.; WANG, L.; LIU, L.; ZHAO, C.; ZHENG, Y. Association mapping of agronomic traits on chromosome 2A of wheat. Genetica, v.137, p.67-75, 2009. DOI: 10.1007/ s10709-009-9351-5.

YU, J.M.; PRESSOIR, G.; BRIGGS, W.H.; VROH BI, I.; YAMASAKI, M.; DOEBLEY, J.F.; MCMULLEN, M.D.; GAUT, B.S.; NIELSEN, D.M.; HOLLAND, J.B.; KRESOVICH, S.; BUCKLER, E.S. A unified mixed-model method for association mapping that accounts for multiple levels of relatedness. Nature Genetics, v.38, p.203-208, 2006. DOI: 10.1038/ng1702.

YU, J.; ZHANG, Y.; ZHU, C.; TABANAO, D.A.; PRESSOIR, G.; TUINSTRA, M.R.; KRESOVICH, S.; TODHUNTER, R.J.; BUCKLER, E.S. Simulation appraisal of the adequacy of number of background markers for relationship estimation in association mapping. Plant Genome, v.2, p.63-77, 2009. DOI: 10.3835/ plantgenome2008.09.0009.

ZHANG, K.; WANG, J.; ZHANG, L.; RONG, C.; ZHAO, F.; PENG, T.; LI, H.; CHENG, D.; LIU, X.; QIN, H.; ZHANG, A.; TONG, Y.; WANG, D. Association analysis of genomic loci important for grain weight control in elite common wheat varieties cultivated with variable water and fertilizer supply. PLoS ONE, v.8, e57853, 2013. DOI: 10.1371/journal. pone. 0057853

ZHANG, Z.; ERSOZ, E.; LAI, C.-Q.; TODHUNTER, R.J.; TIWARI, H.K.; GORE, M.A.; BRADBURY, P.J.; YU, J.; ARNETT, D.K.; ORDOVAS, J.M.; BUCKLER, E.S. Mixed linear model approach adapted for genome-wide association studies. Nature Genetics, v.42, p.355-360, 2010. DOI: 10.1038/ng.546. 